\title{
Transmissão do diagnóstico de câncer sob a perspectiva da observação psicológica
}

Transmission of cancer diagnosis under the perspective of psychological observation

Transmisión del diagnóstico de cáncer bajo la perspectiva de la observación psicológica

Tamires Daiane da SILVA ${ }^{1}$

Débora FOGER $\mathbf{1}^{1}$

Elaine Cristina Gardinal PIZATO²

Paulo Sérgio da Silva SANTOS ${ }^{1}$

${ }^{I}$ Departamento de Cirurgia, Estomatologia, Patologia e Radiologia da Faculdade de Odontologia de Bauru, Universidade de São Paulo, USP 17012-901,Bauru SP, Brasil ${ }^{2}$ Departamento de Psicologia das Faculdades Integradas de Jaú, FIJ 17207-310 Jaú-SP, Brasil

\section{Resumo}

Introdução: A transmissão do diagnóstico de câncer pode gerar ansiedade e nervosismo no indivíduo, por não saber lidar com essa situação, assim, o indivíduo entende como se a morte fosse o único desfecho nessa situação. Objetivo: observar como a pessoa com câncer e seus familiares se comportaram no momento do diagnóstico. Materiais e métodos: Para realizar este estudo, utilizou-se o relato de caso para avaliar como o paciente e seus familiares receberam informações sobre o diagnóstico de câncer. O procedimento utilizado foi a observação não participante, pois com ela foi possível descrever os dados e comportamentos expostos em um determinado momento. Resultados: Por meio da observação, ficou claro o quanto o diagnóstico de câncer sacode a estrutura familiar, uma vez que a doença se refere ao sinônimo de morte. Conclusões: foi possível observar os comportamentos em uma situação difícil, que é receber um diagnóstico de câncer. Com este estudo, os pontos comuns entre o que foi observado e o que a literatura traz sobre a transmissão do diagnóstico de câncer, como medo e ansiedade, foram percebidos.

Descritores: Neoplasias; Diagnóstico Bucal; Psicologia; Observação.

\section{Abstract}

Introduction: The transmission of the diagnosis of cancer can generate in the individual anxiety and nervousness because they do not know how to deal with this situation; thus, the individual understands as if death were the only outcome in the face of this situation. Objetctive: observe how the person with cancer and their relatives behaved at the time of diagnosis. Materials and methods: To perform this study, we used the case report to evaluate how a patient and his / her family members received information about the diagnosis of cancer. The procedure used was observation, since it is possible to describe the data and behaviors exposed at a given moment. Results: Through observation, it was clear how the diagnosis of cancer undermines the family structure, since this disease refers to the synonym of death. Conclusions: It was possible to observe the behaviors before a difficult situation, that is to receive the diagnosis of cancer. With this study, the common points between what was observed and what the literature brings about the transmission of the diagnosis of cancer, such as fear and anxiety, were perceived.

Descriptors: Neoplasms; Diagnosis, Oral; Psychology; Observation.

\section{Resumen}

Introducción: La transmisión del diagnóstico del cáncer puede generar en el individuo ansiedad y nerviosismo por el hecho de no saber lidiar con esa situación; siendo así, el individuo entiende como si la muerte fuese el único desenlace ante esta situación. Meta: observar cómo la persona con cáncer y sus familiares se comportaron en el momento del diagnóstico. Materiales y métodos: Para realizar este estudio, se recurrió al relato de caso para evaluar cómo un paciente y sus familiares recibieron la información del diagnóstico de cáncer. El procedimiento utilizado fue la observación, ya que con ella es posible describir los datos y comportamientos expuestos en un determinado momento. Resultados: Por medio de la observación, quedó claro cuánto el diagnóstico de cáncer sacude la estructura familiar, una vez que esa enfermedad remite al sinónimo de muerte. Conclusiones: Fue posible observar los comportamientos ante una situación difícil, que es recibir el diagnóstico de cáncer. Con ese estudio, se percibió los puntos en común entre lo que fue observado y lo que la literatura trae sobre la transmisión del diagnóstico de cáncer, como el miedo y la ansiedad.

Descriptores: Neoplasias; Diagnóstico Bucal; Psicología; Observación.

\section{INTRODUÇÃO}

Entende-se por câncer um conjunto de células anormais, que se proliferam pelo corpo do indivíduo de forma desenfreada, prejudicando assim, as demais células ${ }^{1}$.

Para obter o diagnóstico de um câncer, são utilizados alguns métodos como a biópsia, punção, exame de sangue, entre outros. Só assim é possível identificar o tipo do tumor, sendo ele benigno ou maligno, e quais tratamentos poderão ser utilizados. Após o diagnóstico, o médico analisará qual a melhor forma de tratamento para determinada pessoa ${ }^{1}$.

Mesmo a medicina tendo evoluído bastante no combate ao câncer, uma das formas mais efetivas de proporcionar a cura ou qualidade de vida ao paciente é o diagnóstico precoce ${ }^{2}$. Infelizmente, o índice de diagnóstico tardio é significativamente grande, tornando-se algo alarmante. Carvalho ${ }^{3}$ (2002) afirma que $60 \%$ dos cânceres existentes são possíveis de serem prevenidos de diversas formas (hábitos alimentares, exames, entre outros), mas para isso, é preciso existir uma política séria voltada para a saúde. Nesse sentido, a Organização Mundial da Saúde $^{4}$ (2007), "declara que $40 \%$ de todo o câncer no mundo poderia ser prevenido por meio de exames".

A transmissão do diagnóstico do câncer pode gerar no indivíduo ansiedade e nervosismo pelo fato de não saber lidar com essa situação; não somente sintomas, mas o paciente pode desenvolver transtornos psicológicos, como a depressão por exemplo. Após o diagnóstico, a dinâmica familiar também pode sofrer mudanças nas relações interpessoais ${ }^{3,5,6}$.

Transmitir ao paciente que ele tem câncer não é algo fácil para os profissionais da saúde devido ao contexto histórico da doença, bem como a forma como é passada essa notícia pode implicar no andamento do tratamento do indivíduo. A comunicação é a principal ferramenta nesse contexto, juntamente da interação e relação entre o profissional e o paciente. $\mathrm{O}$ que não pode acontecer é privar o paciente de receber o diagnóstico da neoplasia, embora ainda existam profissionais e familiares que 
são a favor da omissão da notícia ${ }^{7}$.

Devido a este acontecimento, frente ao diagnóstico de câncer, o indivíduo muitas vezes entende como se a morte fosse o único desfecho; isso torna o entendimento sobre a neoplasia simplista, ou ainda como afirmou Yamaguchi ${ }^{1}$ (p. 21, 2003), “[...] genérico [...]", pois como explicitado à cima, já se faz presente na percepção do ser humano; o termo genérico também remete a ideia de que todas as pessoas apresentarão os mesmos sintomas e desfecho, esquecendo-se que cada pessoa é singular, sendo assim, esses dois aspectos não prevalecerão em todos os indivíduos da mesma forma ${ }^{8}$.

Para o indivíduo lidar melhor com esta situação, poderá recorrer ao profissional da Psicologia; este por sua vez, poderá trabalhar crenças e estigmas em torno da doença, uma vez que essas crenças podem estar enraizadas na sociedade, como por exemplo, ver o câncer como punição pela vida que determinada pessoa levou'. O psicólogo pode atuar também com os familiares dos pacientes que também não sabem lidar com essa situação, e que muitas vezes tem a rotina familiar modificada por inúmeros motivos: como a internação da pessoa que provê o sustento da casa; a mãe que é internada e fica longe do filho pequeno; a criança que tem câncer, entre outros inúmeros fatores ${ }^{2}$. O objetivo desse relato de caso foi o de observar como a pessoa com câncer e seus familiares se comportaram no momento do diagnóstico.

\section{MATERIAL E MÉTODO}

\section{- Procedimentos}

A observação foi realizada no Centro de Pesquisa Clínica da Faculdade de Odontologia de Bauru da Universidade de São Paulo (USP). Logo após o diagnóstico positivo para a neoplasia, os profissionais são responsáveis por encaminhar os pacientes para o Serviço de Orientação e Prevenção do Câncer (SOPC) para a primeira consulta com um oncologista e para o Centro de Pesquisas Clínicas para preparar a boca do paciente para receber o tratamento antineoplásico.

Para coletar os seguintes dados foram observadas as falas bem como as posturas corporais apresentadas por Dona Ester (nome fictício para resguardar a identidade da paciente) e por suas filhas.

\section{- Instrumentos}

Para realizar este estudo de caso, utilizou-se como método a observação não-participante, onde o pesquisador atua como expectador. A observação é um método científico que permite ao psicólogo coletar dados diante de determinada situação. Esses dados têm relação com o fenômeno observado, ou seja, com o comportamento humano, bem como com o meio social em que o indivíduo está inserido. É por meio da observação que o psicólogo consegue identificar o problema e propor a melhor técnica que possa ser utilizada para atingir seu público-alvo ${ }^{10}$.

- Participantes

Participou do estudo a paciente cujo nome fictício será Ester. Ela tem 71 anos, é aposentada. Ester foi diagnosticada com um carcinoma espinoceluar (CEC) de mucosa jugal direita com envolvimento de rebordo alveolar; relatou que fuma desde os 15 anos de idade e atualmente fuma três maços de cigarros por dia (em torno de 60 cigarros); também é etilista. Apesar de Dona Ester estar acompanhada por duas filhas, o diagnóstico foi transmitido diretamente para ela, já que apresentava boas faculdades mentais. Foi explicado a ela e as filhas quais procedimentos deveriam ser realizados a fim de dar início ao tratamento oncológico.

\section{RESULTADOS E DISCUSSÃO}

Os resultados e discussões foram descritos a partir da observação não participante da transmissão do diagnóstico de câncer.

\section{- Observação do atendimento}

Ester foi a primeira pessoa a entrar no consultório odontológico. A paciente entrou sem esboçar qualquer emoção; ao adentrar, cumprimentou a cirurgiã-dentista que fez sua biópsia, após, foi conduzida à cadeira para retirar os pontos do procedimento odontológico (biopsia), para identificar qual o tipo de lesão que havia em sua boca. Em seguida, a filha parou na porta, observou a movimentação e entrou assim que pediram para ela se sentar. Alguns minutos depois, chegou a segunda filha e logo tratou de se acomodar em outra cadeira.

A primeira filha esboçava estar preocupada, nervosa, apreensiva e ansiosa; a segunda filha estava agitada e relatou a irmã: "meu coração está disparado" (sic). A postura da primeira filha estava encurvada e fechada, estava com os olhos vermelhos, aparentando ter chorado antes mesmo de entrar no consultório; em contrapartida, a outra irmã apresentou postura ereta e aberta.

A postura da cirurgiã-dentista foi adequada para o momento em questão. Em sua abordagem, perguntou a paciente como havia passado o final de semana e aos poucos, foi agregando as filhas no diálogo.

Para transmitir a notícia, foi chamada uma docente do departamento de Estomatologia, a qual, ao entrar na sala anunciou que o resultado do exame havia ficado pronto, e neste momento, Ester se pronunciou: "esse é meu medo" (sic). Logo depois, a docente relatou o diagnóstico positivo de câncer. A equipe de cirurgiões-dentistas da Faculdade de Odontologia de Bauru mostrou preocupação em torno do tratamento, fazendo-se assim, o agendamento prévio da primeira consulta ao Serviço de Orientação e Prevenção do Câncer (SOPC), com o oncologista para iniciar o recurso terapêutico; também agendaram o tratamento odontológico no Centro de Pesquisa 
Clínica (CPC), pertencente também a FOB-USP, no objetivo de adequar a condição bucal antes do início do tratamento antineoplásico, para evitar complicações, e colocou a disposição da paciente e familiares o suporte odontológico durante a realização do tratamento do câncer.

Antes e após o diagnóstico observou-se o quanto as filhas de Dona Ester estavam nervosas; já em relação a paciente, esta mostrou-se aparentemente calma. A psicóloga fez-se presente neste momento caso fosse preciso prestar amparo psicológico a paciente e/ou às suas filhas.

As duas filhas que estavam presentes, ouviram atentamente o passo a passo que deveria ser feito para iniciar o quanto antes o tratamento antineoplásico e mostraram-se solicitas com as recomendações.

\section{CONCLUSÃO}

A técnica da observação não-participante mostrou-se eficaz diante do que foi proposto no estudo, pois foi possível observar os comportamentos diante de uma situação difícil, que é receber o diagnóstico de câncer. Por meio da observação, ficou claro o quanto o diagnóstico de câncer abala a estrutura familiar, uma vez que essa doença remete ao sinônimo de morte.

$\mathrm{O}$ aspecto mais relevante visto neste estudo foi tanto a consulta como o apoio que equipe odontológica, junto da psicóloga, deu para essa família, com o agendamento da primeira consulta ao oncologista no SOPC, bem como o cuidado odontológico que será realizado antes, durante e após o tratamento antineoplásico.

\section{AGRADECIMENTOS}

O presente trabalho foi realizado com apoio da Coordenação de Aperfeiçoamento de Pessoal de Nível Superior - Brasil (CAPES) - Código de Financiamento 001.

\section{REFERÊNCIAS}

1. Yamaguchi NH. O câncer na visão da oncologia. In: Carvalho MMMJ. Introdução à Psiconcologia. Campinas: Livro Pleno; 2003.

2. Gimenes MGG. Definição, foco de estudo e intervenção. In: Carvalho MMMJ. Introdução à Psiconcologia. Campinas: Livro Pleno; 2003.

3. Carvalho MMMJ. Psico-Oncologia: história, características e desafios. Psicologia USP. 2002;13:151-66.

4. World Halth Organization (WHO). The World Health Organization's fight against cancer: strategies that prevent, cure and care. 2007.

5. Farinhas GV, Wendling MS, Dellazzana-Zanon LL. Impacto psicológico do diagnóstico de câncer na família: um estudo de caso a partir da percepção do cuidador. Pensando Fam. 2013;17: 111-29.
6. Landskron LMF. Psicooncologia: as descobertas sobre o câncer ao longo da história. In: Hart CFM. Câncer: uma abordagem psicológica. Porto Alegre: AGE; 2008.

7. Silva VCE, Fontão Zago MM. A revelação do diagnóstico de câncer para profissionais e pacientes. Rev Bras Enferm. 2005;58:476-80.

8. Carvalho MMMJ. O sofrimento da dor em câncer. In: Carvalho MMMJ. Introdução à Psiconcologia. Campinas: Livro Pleno. 2003.

9. Scorsolini-Comin F, Vilela e Sousa L, Santos MA. Tornanr-se psicólogo: experiência de estágio de Psico-Oncologia em equipe multiprofissional de saúde. Rev bras orientac prof. 2008;9(2):11325.

10.Donna MF, Matos MA. A necessidade da observação em ciência. In: Donna MF, Matos MA. Ensinando observação: uma introdução. 4. ed. São Paulo: Edicon; 1999.

\section{CONFLITO DE INTERESSES}

Os autores declaram não haver conflitos de interesse.

\section{AUTOR PARA CORRESPONDENCIA}

\section{Tamires Daiane da Silva}

tamires_psico@usp.br
Submetido em 18/04/2019

Aceito em 28/08/2019 